\title{
Guidelines for oral, pharyngeal and laryngopharyngeal surgery during COVID-19 pandemic
}

\author{
Parvez Husain*, Benazeer Husain, Shaheen Khalil Ahmed
}

Department of ENT, Expert ENT and Diagnostic Centre, Barabanki, Uttar Pradesh, India

Received: 27 January 2021

Accepted: 09 March 2021

*Correspondence:

Dr. Parvez Husain,

E-mail: parvez.talkme@gmail.com

Copyright: ( ) the author(s), publisher and licensee Medip Academy. This is an open-access article distributed under the terms of the Creative Commons Attribution Non-Commercial License, which permits unrestricted non-commercial use, distribution, and reproduction in any medium, provided the original work is properly cited.

\begin{abstract}
The pandemic due to the new respiratory infection known as coronavirus 2019 disease (COVID-19), caused by the SARS-CoV-2 virus, has triggered an unprecedented disruption in the normal activity of ENT, oral, and pharyngeal surgery departments worldwide, delaying routine patient care and elective surgical interventions. ENT, oral, and pharyngeal surgeons are one of the healthcare groups with the highest risk of nosocomial infection because of the close contact that occurs with asymptomatic and symptomatic patients with SARS-CoV-2 infection through the oral cavity and laryngopharynx. The purpose of this document has been to update the available evidence for the safe and effective management and treatment in elective and emergency surgeries, and hospitalization, while minimizing as much as possible the risk of infection for the ENT, oral, and laryngopharyngeal surgeon, health workers and patients. This document aims to clarify the most significant aspects and develop a common protocol for the surgical management of patients with COVID-19 in ENT, oral, and maxillofacial surgery during the acute stage of spread and subsequent control of the pandemic in our country.
\end{abstract}

Keywords: COVID-19, Oral, Laryngopharyngeal, Pandemic, Guidelines

\section{INTRODUCTION}

The epidemic of coronavirus disease 2019 (COVID-19), originating in Wuhan, Hubei province, China, and rapidly spreading to other provinces of China and 190 other countries, was declared a global pandemic by WHO on March 9, 2020, becoming a "public health emergency of international concern". Patients who are COVID-19 positive are the main source of infection, the asymptomatic ones are extremely contagious, with a strong infectivity in the incubation period ranging from 1 to 14 days. The person-to- person transmission routes of 2019-nCoV included direct transmission, such as coughing, sneezing, droplet inhalation, and contact transmission, such as contact with oral, nasal, and eye mucous membranes. The faecal-oral route remains to be determined. Infection control measures are necessary to prevent the virus from further spreading and to help control the epidemic situation. ${ }^{1}$ The risk of infection during the diagnosis and treatment of oral and oropharyngeal diseases was also quickly assessed, suspending non-urgent outpatient oral treatments and maintaining the main emergencies of the oropharyngeal area represented by trauma, malignant neoplasms, and infections, which require timely treatment.

Research shows that there is a high viral load in the nasopharyngeal and oropharyngeal area. ${ }^{2}$ This makes managing patients with pathologies that require oral and laryngopharyngeal surgery to be a major concern especially with the use of cautery, laser, and drills in these surgeries causing aerosolisation of COVID-19. Bioaerosol transmission occurs while doing intubation and cardiopulmonary resuscitation. Fomite transmission is also possible The virus is most contagious when people are asymptomatic: although spread can occur even before symptoms appear. Airborne transmission of SARS-CoV-2 can occur during medical procedures that generate aerosols ("aerosol generating procedures"). ${ }^{3}$ 
Every healthcare worker and specialty is at increased risk of exposure as well as acting as a source of transmission to the community. ${ }^{4}$ ENT, oral and laryngopharyngeal surgery is the branch that extends from a life savior of trauma and cancer patients to an artist for sculpturing beautiful faces out of anomalies. We should also consider the vicinity of our area of interest to airway that makes us highly vulnerable to COVID-19 infection. Our surgical practice is also not spared from the effects of this gigantic, invisible enemy that all of us are facing, i.e., novel corona virus called as COVID-19 pandemic.

Operating room (OR) is the heart of every surgical specialty, and in such difficult times, it is important to take very stringent actions and follow certain guidelines without any loop holes. Hence, it is of utmost importance for us to educate ourselves and act tactfully to serve the patients as well as protect the community and ourselves. This article exclusively answers the dilemma an ENT and oropharyngeal surgeon will be facing while approaching the patients during COVID-19 pandemic.

Keeping all this in mind the procedures which need instrumentation of upper airway should be done only in cases of emergency or if absolutely necessary, COVID-19 testing should be given importance. ENT and oral surgeons should be aware of utmost importance of effective use of full or enhanced personal protective equipment (PPE) during diagnostic and therapeutic procedures dealing the oral and aero-digestive tract. Enhanced PPE includes N95 mask with a face shield or powered air-purifying respirator (PAPR), surgical cap, surgical gown and double gloves. Operating room (OR) protocols and alteration in practice required during and after the global pandemic of COVID-19 shall be divided into seven parts for ease of expression, namely: case evaluation and selection; pre-operative preparation and arrangement of OR; the day of surgery; patient preparation; surgical and anaethesia protocols; postoperative care of OR; and postoperative patient care.

\section{CASE EVALUATION AND SELECTION}

A questionnaire has to be used to screen patients both by phone and at the time of admission.

This procedure is important because the patients with oral and laryngopharyngeal emergencies often have symptoms such as fever and fatigue due to acute stress and inflammation. Therefore, a first evaluation is essential to accurately identify suspicious or high-risk patients.

All patients undergoing oral or laryngopharyngeal surgery should be discussed with the assisting surgeon, anaesthetist, scrub nurse and other health care staff and should be divided into: elective, emergency surgery. If delaying surgery is unlikely to cause harm then review every 3 months or sooner if the patient develops any change in symptoms.
Patient selection has to be done very carefully, and only emergencies like oral and laryngeal trauma, infections and essential elective oral cancer surgeries need to be performed during this pandemic. These surgeries cannot be postponed and need special attention as time plays a very important factor in healing and final outcome. Elective surgeries need to be deferred and, if at all, want to plan, patient should be in quarantine for 14 days before planned for elective procedure. ${ }^{5}$

Al-Muharraq et al suggested testing recommendation for COVID-19 (SARS-CoV-2) in patients planned for surgery. ${ }^{6}$ We should try to understand the availability and the types of the test available for COVID-19 disease in India which at the moment is available only for the symptomatic patient. There are two categories of the tests that are available: nucleic acid amplification test for viral RNA using polymerized chain reaction (PCR) and antibody detection test via serology. ${ }^{7}$

\section{Pre-operative preparation and arrangement of $O R$}

Test should be done of all potential admissions for COVID-19. The patients must have self-isolate themselves for at least seven days before admission. ${ }^{8,9}$ Patients who have no symptoms suggestive of COVID-19 infection, have been isolated for seven days and have a negative COVID-19 test should be admitted to a COVID free facility (ward) and operated on in a COVID free theatre. After seven days of isolation patient is admitted 48 hours prior to surgery. The second test is done at the time of admission, if the test is negative high resolution computed tomography (HRCT) of chest is done to exclude any false negative test result. HRCT chest positive findings included ground-glass opacity, local patchy shadowing, bilateral patchy shadowing, or interstitial abnormalities.

As PCR testing may only be around $75 \%$ sensitive the theatre team should still wear full PPE as for COVID positive patients as all these procedures are aerosol generating. ${ }^{9}$

Patients who are COVID-19 positive should be admitted to a COVID facility and operated on in a COVID theatre wearing full PPE as all these procedures are aerosol generating. 9

\section{ARRANGEMENT OF OR}

\section{Equipments}

Minimal fomite-bearing surfaces in OR (equipments, extra medications and surgical material not to be used in the ongoing surgery to be removed from OR and kept separately in the store room).

All the surfaces of equipment like OR table, anesthesia work station, monitors, electrocautery and motor drills to be covered with plastic sheets. The sheets to be changed after every patient. 
Intercom to be installed in OR connected to every department of hospital for better and quicker communication.

It is recommended to use OR having laminar air flow system and HEPA filter ( 0.1 micron efficiency) is recommended relative to surrounding air. ${ }^{10} \mathrm{~A}$ high frequency of air changes ( 25 per hour) rapidly reduces viral load within the OR.

Prefer not to use air conditioners inside OR and in areas of possible air contamination.

At the entry of OR and exit, there should be a no touch sensor-based sanitizer dispenser.

High-volume suction to be one-third prefilled with povidone-iodine solution. The povidone-iodine solution is believed to have virucidal effects and reduces viral load in aerosols produced by suction machine.

A high-quality heat and moisture exchanging filter (HMEF) rated to remove at least $99.97 \%$ of airborne particles of 0.3 microns or greater should be placed between the face mask and the breathing circuit or between the face mask and the reservoir bag as applicable. Insert bacterial viral filter to the expiratory limb of the breathing circuit apart from the heat and moisture exchanger (HME). ${ }^{11}$

\section{OR staff}

Minimal staff in OR. Only experts to perform intubation and surgery. This reduces aerosolization of the bronchial secretions and also reduces the surgical time.

No observers allowed inside the OR. This reduces the exposure of other healthcare personnel to any infection.

During intubation and extubation, only anesthesia team inside OR. The surgical team has to wait in the waiting area and allowed to enter after 21 min of completion of intubation. The contaminated aerosols settle down from the air during this period, thus reducing exposure to the surgeon. ${ }^{12}$

During surgery, only surgical team to be present inside the OR. Anesthesia team to monitor patient from waiting to be sitting behind transparent barrier. This reduces the exposure time for the anesthetists.

\section{DAY OF SURGERY}

Irrespective of the PCR test results all the operation theatre (OT) staff should wear full or enhanced PPE which includes N95 mask with a face shield or powered airpurifying respirator (PAPR), surgical cap, surgical gown and double gloves. OT staff should be pre-assigned and once entered into the OT with enhanced PPE should not leave the room till the procedure ends or due to some special circumstances. Also, the staff outside the OT should not enter the OT.

\section{PATIENT PREPARATION}

Gargling with povidone-iodine (at a concentration of 0.23 $1 \%$ ) for 1 minute 1-2 hours prior to surgery will reduce the viral load in the pharynx by at least $4 \mathrm{x} \quad \log 10$ (corresponding to a reduction in viral titre of $\geq 99.99 \%$ for all coronaviruses tested. ${ }^{13,14}$ Povidone iodine, has been used as a gargle for mucositis and nasal rinse for recalcitrant sinusitis with no negative effect on olfaction, mucociliary clearance and improvement in mucositis. ${ }^{15-17}$ If the patient is iodine intolerant then we would suggest $0.5 \%$ hydrogen peroxide. ${ }^{18}$

Irrespective of COVID screening results, it is advisable to treat all patients undergoing aerosol generating procedures as COVID-19 positive, and the whole theatre team should wear PPE as per current national guidelines. ${ }^{9}$

Induction of anaesthesia and intubation to be done in theatre and not in anaesthetic room to minimise potential contamination. While intubating the patient the surgical team should wait outside the operation theatre, postintubation for 21 minutes. ${ }^{19}$

Oral and throat pack removal is aerosol generating and, therefore, recommend not using throat packs where it is safe to do so.

\section{SURGICAL AND ANAESTHESIA PROTOCOLS}

\section{Surgical protocols}

The following surgical protocols were followed: all surgeons to use positive air pressure respirators (PAPR) in addition to suggested PPE; surgical team to wait in waiting area during intubation and extubation and to enter 21 minutes after procedure; pack oral and nasal cavity with bio-occlusive dressing before taking an extraoral approach; avoid use of cautery, drills, and forceful irrigation unless unavoidable; communication and improved team approach to avoid injury to protective gear; use of povidone-iodine (10\% solution in 1:9 dilution) and hydrogen peroxide $(3 \%)$ in irrigation solution to minimize viral load in aerosols. ${ }^{19}$

\section{Anesthesia protocols}

During intubation and extubation the aerosolization and droplet transmission of the COVID-19 virus are important hazards in the environment of the operating theaters, especially with procedures such as endotracheal intubation and tracheostomy. ${ }^{20}$

At the time of intubation and extubation, only required auxiliary staff should be allowed in OR. 
All the staff in the OR must wear PPE (a fit-tested N95 mask, face shield or goggles, hazmat suits and gloves and shoe covers).

The attempt will be made to extubate all cases in the OR suite itself at the end of surgery.

Avoid awake fiber optic intubation and preferably use video laryngoscope. ${ }^{21}$

Preoxygenate with minimal gas flow possible, i.e. less than $6 \mathrm{~L}$ per min, ensure good seal with face mask; give fentanyl slowly, in small aliquots if required to reduce coughing. ${ }^{22}$

Utilize rapid sequence induction to reduce the need for mask ventilation.

Give anti-emetics to minimize vomiting.

\section{Maintenance under anesthesia}

The following conditions were maintained during anaesthesia: minimize tube and circuit disconnection; use a closed suctioning system if available; place the ventilator on standby whenever a circuit disconnection is required, such as tube repositioning; restart mechanical ventilation only after the circuit has been reconnected/closed; employ lung protective mechanical ventilation strategies by maintaining tidal volumes of $5-6 \mathrm{ml} / \mathrm{kg}$; and increase respiratory rate to maintain minute ventilation, and keep peak airway pressure below $30 \mathrm{mmHg} .{ }^{23}$

\section{Postoperative care of $\mathrm{OR}$}

The following post-operative care of OR was taken: every surface of OR to be considered as contaminated after surgery; once the patient has been shifted to the recovery room, surgical and anesthesia staff to remove PPE and leave the OR; all the plastic sheets to be removed and disposed in disinfectant containing bin; all the surfaces to be sprayed with sodium hypochlorite $(1 \%)$; alcohol-based spray to be used on monitors; both HME filters and the soda lime should be changed; OR to be fumigated and sealed for 24 hours, but formaldehyde is a carcinogen and affects lungs which are primary target organs for COVID19 infection; and fogging can be a good alternative to fumigation as it generates fog or mist formed by ultralowvolume (ULV) uniform submicron-size liquid particles (dry fog) and possesses no risk to lungs. Various chemicals are used for fogging procedure like glucoprotamine, glutaraldehyde, hydrogen peroxide and silver nitrate.

\section{Postoperative patient care}

The following post-operative care was taken: minimise the post-operative stay to reduce the risk of hospital acquired COVID; and consider telephone/video clinic follow up appointments unless clinical examination is considered essential. $^{24}$
All the elective routine surgeries are deferred till the COVID-19 pandemic gets over. If the surgery is posted then the patient and family members are asked to selfquarantine till the surgery. If the tests are negative then COVID-19 protocol is followed. If the tests are positive, then it is better to defer the case and try alternative treatment unless and until it is a life threatening condition.

\section{DISCUSSION}

COVID-19 is a pandemic, health care staff, ENT, and oral and maxillofacial surgeons are at high risk of getting infected. Although many surgical cases are deferred, still crucial life-threatening cases need to get operated. As an ENT and oral and pharyngeal surgeon, it is our duty to maintain the safety of ourselves, our auxiliary staff and our patients from cross-contamination. Optimization and upgradation of infection control protocol in operating room during the COVID-19 pandemic are mandatory.

Confirmed modes of viral transmission are primarily contact with contaminated environmental surfaces and aerosolization. Microorganisms that include the novel corona virus are not visible to naked eye and hence requires a very stringent approach to tackle.

Pederson et al conducted a video audit of operating room procedures and suggested deep surface cleaning to be augmented with UV light disinfection. ${ }^{25}$ This can be added to OR protocols along with chemical disinfection and fumigation.

Australian society of anesthesia advises that extubation should take place in the operating theater, and it is recommended that recovery of the patient also take place in the operating theater if resources allow.Based on the surgeon general's advice, all elective procedures should be currently halted. Surgeries at this point should only be considered for emergent/urgent cases. In the minority of cases where surgery cannot be avoided, a protocol should be followed i.e. COVID-19 test, high resolution computed tomography (HRCT) chest, and wearing full or enhanced PPE during diagnostic and therapeutic procedures dealing the aero-digestive tract.

\section{CONCLUSION}

We suggest oral and laryngopharyngeal surgery to be very safe for staff and patients. Accurate planning before the surgery and its execution as planned is recommended, and the surgeries are to be done by experienced surgeons.

We recommend the need to augment our strategies to reduce cross-contamination and transmission of COVID19 pandemic. Considering the current health crisis caused by COVID-19, the above guidelines are recommended for performing oral and laryngopharyngeal surgeries.

Funding: No funding sources

Conflict of interest: None declared

Ethical approval: Not required 


\section{REFERENCES}

1. Zhang T, Liu T, Yu L. Instructions for management of oral andmaxillofacial trauma during prevention and control stage of the novelcoronavirus infection. Chin J Oral Maxillofac Surg. 2020;2:100-4.

2. Zou L, Ruan F, Huang M, Liang L, Huang H, Hong $Z$, et al. SARS-CoV-2 Viral Load in Upper Respiratory Specimens of Infected Patients. N Engl J Med. 2020;382(12):1177-9.

3. Advice on the use of masks in the context of COVID19. Interim guidance. Geneva: World Health Organization. 2020. Available at: https://www .who.int/publications/i/item/advice-on-the-use-ofmasks-in-the-community-during-home-care-and-inhealthcare-settings-in-the-context-of-the-novelcoronavirus-(2019-ncov)-outbreak. Accessed on 22 January 2021.

4. Lancet T. COVID-19: protecting health-care workers. The Lancet. 2020;395:922.

5. Lei S, Jiang F, Su W et al (2020) Clinical characteristics and outcomes of patients undergoing surgeries during the incubation period of COVID-19 infection. E Clin Med. 2020;21:100331.

6. Al-Muharraqi MA (2020) Testing recommendation for COVID- 19 (SARS-CoV-2) in patients planned for surgery-continuing the service and 'suppressing' the pandemic. Br J Oral Maxillofac Surg. 2020;58(5):503-5.

7. Patel R, Babady E, Theel ES, Storch GA, Pinsky BA, St George K, Smith TC, Bertuzzi S. Report from the American Society for Microbiology COVID-19 International Summit, 23 March 2020: Value of Diagnostic Testing for SARS-CoV-2/COVID-19. mBio. 2020;11(2):e00722-20.

8. Academy of Medical Royal Colleges. Clinical guide for the management of essential cancer surgery for adults during the coronavirus pandemic. 2020. Available at: https://www.england.nhs.uk /coronavirus/wp-content/uploads/sites/52/2020/04

/C0239- Specialty-guide-Essential-Cancer-surgeryand-coronavirus-v1-70420.pdf 5. Accessed on 22 January 2021.

9. Available at: https://www.gov.uk/government/ publications/wuhan-novel-coronavirus-infectionprev ention-and-control. Accessed on 22 January 2021.

10. Schentag JJ, Akers C, Campagna P, Chirayath P. SARS: clearing the air. Institute of Medicine (US) Forum on Microbial Threats; Knobler S, Mahmoud A, Lemon S, et al, editors. National Academies Press, Washington. 2004.

11. Wilkes AR. Heat and moisture exchangers and breathing system filters: their use in anaesthesia and intensive care. Part 1: history, principles and efficiency. Anaesthesia. 2011;66:31-9.

12. AO CMF. 2020. Available at: https://aocmf3. aofoundation.org/. Accessed on 26 April 2020.

13. Geller C, Varbanov M, Duval RE. Human coronaviruses: insights into environmental resistance and its influence on the development of new antiseptic strategies. Viruses. 2012;4:3044-68.

14. Eggers M, Koburger-Janssen T, Eickmann M, Zorn J. In Vitro Bactericidal and Virucidal Efficacy of Povidone-Iodine Gargle/Mouthwash Against Respiratory and Oral Tract Pathogens. Infect Dis Ther. 2018;7(2):249-59.

15. Kanagalingam J, Chopra A, Hong M, Ibrahim W, Villalon A, Lin J. Povidone-iodine for the management of oral mucositis during cancer therapy. Oncol Rev. 2017;11(2).

16. Panchmatia R, Payandeh J, Al-Salman R, Kakande E, Habib A, Mullings W, et al. The efficacy of diluted topical povidone-iodine rinses in the management of recalcitrant chronic rhinosinusitis: a prospective cohort study. Eur Arch Oto-Rhino-Laryngol. 2019;276(12):3373-81.

17. Rezapoor M, Nicholson T, Tabatabaee R, Chen A, Maltenfort M, Parvizi J. PovidoneIodine-Based Solutions for Decolonization of Nasal Staphylococcus aureus : A Randomized, Prospective, Placebo-Controlled Study. J Arthroplast. 2017;32(9):2815-9.

18. Kampf G, Todt D, Pfaender S, Steinmann E. Persistence of coronaviruses on inanimate surfaces and their inactivation with biocidal agents. J Hospital Infect. 2020;104(3):246-51.

19. Appendix B. Air guidelines for environmental infection control in health-care facilities, U.S, HHS. 2003. Available at: https://www.cdc.gov/infection control/guidelines/environmental/appendix/air.html. Assessed 20 April 2020.

20. SIAARTI-Covid 19-Airway management. Available at: https://www.esaic.org/esa-news/covid19-airway-management/. Accessed on 30 April 2020.

21. Singh N, Rao PB, Mishra N. Video laryngoscopy in COVID-19 patients: a word of caution. Can J Anaesth. 2020;67(9):1291.

22. Effect of Fentanyl on Coughing and Recovery After Anesthesia With an LMA Laryngeal Mask Airway for Airway Management- Full Text ViewClinicalTrials.gov. Available at: https://clinicaltrials .gov/ct2/show/NCT01368809. Accessed 30 April 2020.

23. Levin MA, McCormick PJ, Lin HM. Low intraoperative tidal volume ventilation with minimal PEEP is associated with increased mortality. $\mathrm{Br} \mathrm{J}$ Anaesth. 2014;113:97-108.

24. Rajan N, Joshi GP. The COVID-19: Role of Ambulatory Surgery Facilities in This Global Pandemic. Anesth Analg. 2020.

25. Pedersen A, Getty Ritter E, Beaton M, Gibbons D. Remote video auditing in the surgical setting. AORN J. 2017;105:159-69.

Cite this article as: Husain $\mathrm{P}$, Husain $\mathrm{B}$, Ahmed SK. Guidelines for oral, pharyngeal and laryngopharyngeal surgery during COVID-19 pandemic. Int J Otorhinolaryngol Head Neck Surg 2021;7:701-6. 
ANNEXURE - 1

\section{Questionnaire}

\begin{tabular}{|ll|}
\hline S. no. & Questions \\
\hline $\mathbf{1}$ & $\begin{array}{l}\text { Do you have fever or have you experienced fever within the past } 14 \text { days? Yes or no } \\
\text { Have you experienced a recent onset of respiratory problems, such as a cough or difficulty in breathing within } \\
\text { the past 14 days? Yes or no }\end{array}$ \\
\hline $\mathbf{3}$ & $\begin{array}{l}\text { Have you, within the past } 14 \text { days, travelled to risk areas or visited neighbourhoods with documented 2019- } \\
\text { nCoV transmission? Yes or no }\end{array}$ \\
\hline $\mathbf{4}$ & $\begin{array}{l}\text { Have you come into contact with a patient with confirmed 2019-nCoV infection within the past } 14 \text { days? Yes } \\
\text { or no }\end{array}$ \\
\hline $\mathbf{5}$ & $\begin{array}{l}\text { Have you recently participated in any gathering, meetings, or had close contact with many people you arenot } \\
\text { acquainted with? Yes or no }\end{array}$ \\
\hline
\end{tabular}

